\title{
Parameter Estimation and Image Reconstruction of Rotating Targets with Vibrating Interference in the Terahertz Band
}

\author{
Qi Yang ${ }^{1} \cdot$ Bin Deng $^{1} \cdot$ Hongqiang Wang ${ }^{1}$ - Yuliang Qin ${ }^{1}$
}

Received: 20 November 2016 / Accepted: 26 April 2017 /

Published online: 10 May 2017

(C) The Author(s) 2017. This article is an open access publication

\begin{abstract}
Rotation is one of the typical micro-motions of radar targets. In many cases, rotation of the targets is always accompanied with vibrating interference, and it will significantly affect the parameter estimation and imaging, especially in the terahertz band. In this paper, we propose a parameter estimation method and an image reconstruction method based on the inverse Radon transform, the time-frequency analysis, and its inverse. The method can separate and estimate the rotating Doppler and the vibrating Doppler simultaneously and can obtain high-quality reconstructed images after vibration compensation. In addition, a $322-\mathrm{GHz}$ radar system and a $25-\mathrm{GHz}$ commercial radar are introduced and experiments on rotating corner reflectors are carried out in this paper. The results of the simulation and experiments verify the validity of the methods, which lay a foundation for the practical processing of the terahertz radar.
\end{abstract}

Keywords Terahertz · Rotating targets · Vibrating interference · Inverse Radon transform · Parameter estimation $\cdot$ Image reconstruction

\section{Introduction}

Micro-motion refers to rotation or precession or other high order motions in addition to mass translation of the targets, and it is a very popular phenomenon in nature and our daily life, such as respiration and heartbeat of human, the vibration of the engines, and the precession of the warheads. Micro-motion may induce additional frequency modulations on the returned signal which generate sidebands about the target's Doppler frequency, called the micro-Doppler

Bin Deng

dengbin_nudt@163.com

1 College of Electronic Science and Engineering, National University of Defense Technology,

Changsha 410073, China 
effect, and it extends the traditional feature extraction and target recognition fields [1]. Rotation is a very important micro-motion form of radar targets, such as the helicopter rotors and the rotating antennas. Detection, measurement and imaging of these rotating targets have important applications in both military and civilian fields, and many researches have been conducted in recent years [2-4].

In practice, however, rotation of the targets is always accompanied with the vibrating interference in many cases, and it will significantly affect the parameter estimation and image reconstruction if the vibrating Doppler reaches a certain degree. Compared with rotation of the targets, the vibrating interference usually owns a higher frequency and smaller amplitude. In synthetic aperture radar and inverse synthetic aperture radar imaging fields, the high frequency vibration of the platforms or the targets is often regarded as an interference signal, especially when the carrier frequency is comparatively high, and several compensation methods are proposed in recent years [5,6]. However, monitoring of the vibrating interference has some value in the specific applications $[7,8]$. For instance, the vibration parameter estimation of the rotating helicopter rotors or the antennas is helpful to analyze the stability of the rotating targets. In fact, micro-Doppler is associated with the movement of the targets and the carrier frequency of the radar system simultaneously just like Doppler. The relatively high carrier frequency of the terahertz radar system makes the micro-Doppler effect more remarkable than that in the microwave band and makes the monitoring of the vibrating interference possible [9, 10].

Aiming at this problem, we propose a parameter estimation method and an image reconstruction method based on the inverse Radon transform, the time-frequency analysis, and its inverse in this paper, and experiments based on a terahertz radar system and a $25-\mathrm{GHz}$ commercial radar are carried out. The paper is organized as follows: the theoretical model of the rotating targets with vibrating interference is established in Section 2. In Section 3, the parameter estimation and image reconstruction methods are proposed and procedures are given in detail. In Section 4, the parameter estimation method is applied to experimental data of a terahertz radar system, and the parameters of rotation and vibrating interference are estimated accurately; additional high-quality images are reconstructed through the methods described in this paper. The conclusions are presented in the last section.

\section{The Theoretical Model and Simulation Scenario}

\subsection{The Theoretical Model}

Generally, the motion of the whole target can be inferred from motions of several scattering centers on it. Since the projection of rotation on the radar slight can be considered as a simple harmonic motion, the motion model of scattering centers on a rotating target can be expressed as

$$
r_{k}(t)=R_{k} \sin \left(2 \pi f_{r k} t+\varphi_{k}\right), \quad k=1,2 \ldots N
$$

where $R_{k}$ and $f_{r k}$ are the micro-motion amplitude and the frequency of the rotating scattering center $k$, respectively, and $\varphi_{k}$ is its initial phase that characterizes the relationship between radar slight and the initial position of the scattering center. Assuming that the transmitting 
signal is a simple-frequency signal with carrier frequency $f_{c}$, and then the echo signal of the rotating target is as follows:

$$
s_{r}(t)=\sum_{k=1}^{N} \sigma_{k}(t) \exp \left(j 2 \pi f_{c}\left(t-\tau_{k}\right)\right)
$$

where $\sigma_{k}$ is the modulation amplitude of the scattering center $k, \tau_{k}=2 r_{k}(\mathrm{t}) / c$ is the echo delay, and $c$ is the speed of light. The baseband signal after mixer can be written as

$$
s_{b}(t)=\sum_{k=1}^{N} \sigma_{k}(t) \exp \left(-j \frac{4 \pi f_{c} R_{k}}{c} \sin \left(2 \pi f_{r k} t+\varphi_{k}\right)\right)=\sum_{k=1}^{N} \sigma_{k}(t) \exp \left(j \Phi_{k}\right)
$$

According to the definition of Doppler, the micro-Doppler of the rotating scattering center is

$$
f_{k}(t)=\frac{1}{2 \pi} \frac{d \Phi_{k}}{d t}=-\frac{4 \pi f_{r k} f_{c} R_{k}}{c} \cos \left(2 \pi f_{r k} t+\varphi_{k}\right), \quad k=1,2 \ldots N
$$

Therefore, it is clear that the micro-Doppler of a rotating scattering center on the target is sinusoidal modulation and its maximum value depends on the carrier frequency, the amplitude, and the frequency of rotation. Meanwhile, the vibrating interference is harmonic in general, which can be shown as follows:

$$
r_{v k}(t)=R_{v k} \sin \left(2 \pi f_{v k} t+\varphi_{v k}\right), \quad k=1,2 \ldots N
$$

where $R_{v k}, f_{v k}$, and $\varphi_{v k}$ represent the amplitude, the frequency, and the initial phase of the vibrating interference of the targets, respectively. Therefore, Eqs. (1), (3), and (4) should be rewritten as follows when considering the vibrating interference of the target (we have assumed that the amplitude and the frequency of the vibrating interference are constant during rotating.):

$$
\begin{gathered}
r_{k}(t)=R_{k} \sin \left(2 \pi f_{r k} t+\varphi_{k}\right)+R_{v k} \sin \left(2 \pi f_{v k} t+\varphi_{v k}\right), \quad k=1,2 \ldots N \\
s_{b}(t)=\sum_{k=1}^{N} \sigma_{k}(t) \exp \left(-j \frac{4 \pi f_{c} R_{k}}{c} \sin \left(2 \pi f_{r k} t+\varphi_{k}\right)-\frac{4 \pi f_{c} R_{v k}}{c} \sin \left(2 \pi f_{v k} t+\varphi_{v k}\right)\right) \\
f_{k}(t)=-\frac{4 \pi f_{r k} f_{c} R_{k}}{c} \cos \left(2 \pi f_{r k} t+\varphi_{k}\right)-\frac{4 \pi f_{v k} f_{c} R_{v k}}{c} \cos \left(2 \pi f_{v k} t+\varphi_{v k}\right), \quad k=1,2 \ldots N
\end{gathered}
$$

It can be seen from Eq. (8) that an extra Doppler term is brought by the vibrating interference and it will cause difficulty to the parameter estimation and imaging. In the terahertz band, the rotating Doppler and the vibrating interference Doppler are far greater than those in the microwave band because of the relatively high carrier frequency, which will make the difficulty more remarkable. However, at the same time, the sensitivity of Doppler in the terahertz band will make the measurement of vibration possible. For example, the vibrating 
Fig. 1 The simulation scenario. This figure shows the micromotion parameters and their basic geometric relationships of the rotating scattering centers

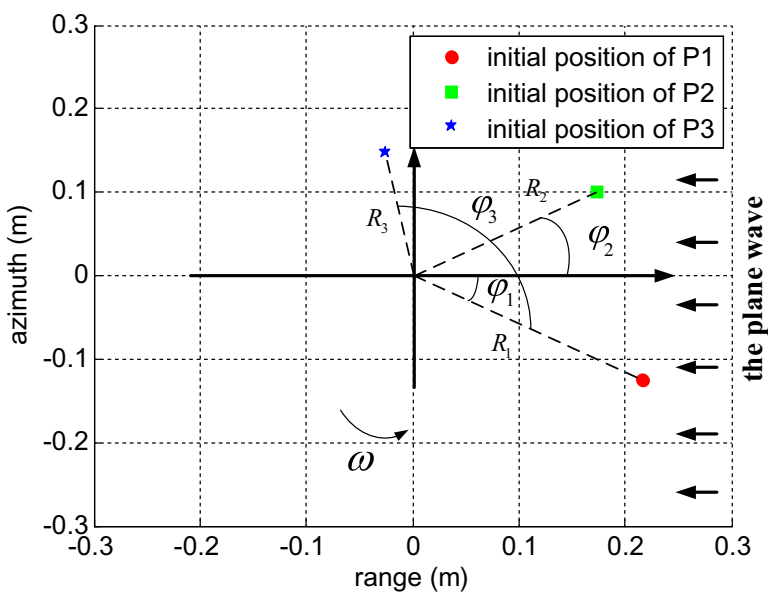

frequency and amplitude of a rotating target is $5 \mathrm{~Hz}$ and $1 \mathrm{~mm}$, respectively, and then the extra Doppler is about $2.1 \mathrm{~Hz}$ when the carrier frequency is $10 \mathrm{GHz}$, and it will reach $67 \mathrm{~Hz}$ at $330 \mathrm{GHz}$. A 2.1-Hz extra Doppler is often difficult to measure in the microwave band, but things will change in the terahertz band.

\subsection{The Simulation Scenario}

A simulation scenario including three rotating scattering centers is shown in Fig. 1, and the time-frequency distributions are shown in Fig. 2. In simulation, the carrier frequency is $322 \mathrm{GHz}$. The rotation period is $12 \mathrm{~s}$, corresponding to an angular velocity of $5 \mathrm{r} / \mathrm{min}$. The rotation amplitudes of $\mathrm{P} 1, \mathrm{P} 2$, and $\mathrm{P} 3$ are $0.25,0.2$, and $0.15 \mathrm{~m}$, and their initial phases are $-30^{\circ}, 30^{\circ}$, and $100^{\circ}$, respectively. The amplitude and frequency of the vibrating interference are $1 \mathrm{~mm}$ and $2 \mathrm{~Hz}$.

Owing to the ability of mapping sinusoidal curves in the original image to peaks in the parameter space, inverse Radon transform in the image reconstruction field is commonly used in parameter estimation and imaging of micro-motion targets [11-13]. For the sinusoidal curves in the time-frequency distribution in Fig. 2a, the focusing performance of inverse

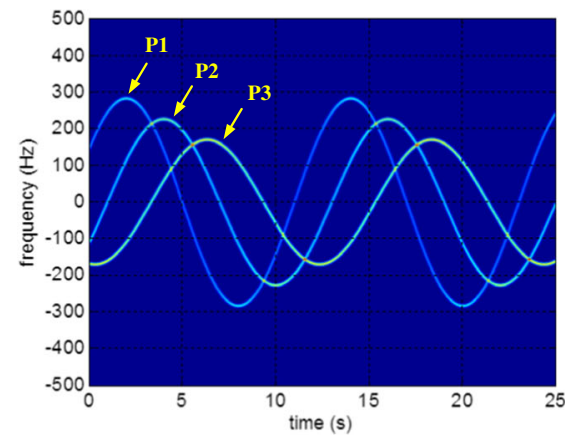

(a) Rotating without vibrating interference

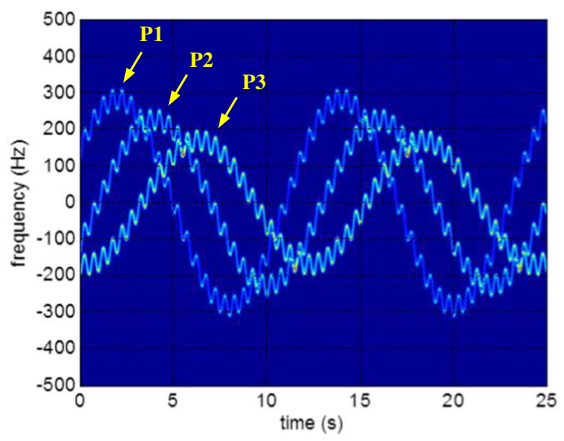

(b) Rotating with vibrating interference

Fig. 2 The time-frequency distributions of the rotating scatter centers. a Rotating without vibrating interference. b Rotating with vibrating interference 


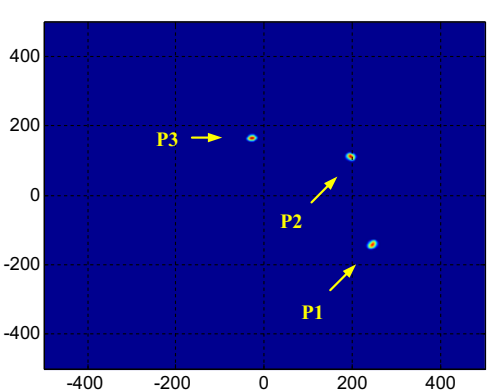

(a) Reconstructed image without vibrating interference

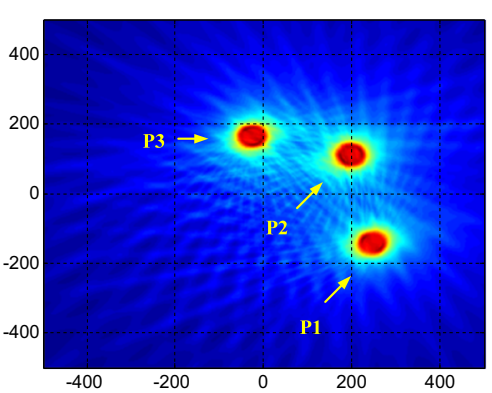

(b) Reconstructed image with vibrating interference

Fig. 3 The inverse Radon transform of Fig. 2. a Reconstructed image without vibrating interference. b Reconstructed image with vibrating interference. The inverse Radon transform results in this situation can be seen as the reconstructed images of the rotating scatter centers, and the defocusing phenomenon in $\mathbf{b}$ is due to the existing of the vibrating interference

Radon transform is extremely good (Fig. 3a), and the parameters of the sinusoidal curves can be derived from positions of peaks in the parameter space. However, the inverse Radon transform of Fig. $2 b$ is severely defocused because of the extra Doppler term resulting from the vibrating interference of the targets (Fig. 3b). Therefore, it is impossible to obtain the parameters accurately by the inverse Radon transform in such a situation.

\section{Parameter Estimation and Image Reconstruction}

\subsection{The Parameter Estimation Method}

In consideration of the vibrating interference, it is difficult to estimate the micro-motion parameters through the inverse Radon transform directly. Therefore, a proper signal separation is necessary. The separation method proposed in this paper contains two aspects: one is the separation of each scattering center through inverse Radon transform and the other is the separation of rotation and vibration depending on their frequency difference. In order to have a thorough understanding of the defocusing reason in Fig. 3b, a simple derivation of the inverse Radon transform of Eq. (8) is shown below.

$$
\begin{aligned}
& g(x, y) \\
& =\sum_{k=1}^{N} \int_{-\infty}^{\infty} \int_{-\infty}^{\infty} \int_{-\infty}^{\infty} \delta\left(\rho-f_{k}(t)\right) \cdot e^{-j 2 \pi \rho v} d \rho \cdot e^{j 2 \pi\left(k_{x} x+k_{y} y\right)} d k_{x} d k_{y} \\
& =\sum_{k=1}^{N} \int_{-\infty}^{\infty} \int_{-\infty}^{\infty} \int_{-\infty}^{\infty} \delta\left(\rho-f_{r k-\max } \sin \left(2 \pi f_{r k} t+\varphi_{k}\right)+f_{v k-\max } \sin \left(2 \pi f_{v k} t+\varphi_{v k}\right)\right) \cdot e^{-j 2 \pi \rho v} d \rho \cdot e^{j 2 \pi\left(k_{x} x+k_{y} y\right)} d k_{x} d k_{y} \\
& =\sum_{k=1}^{N} \int_{-\infty}^{\infty} \int_{-\infty}^{\infty} e^{-j 2 \pi v f_{v k-\max } \sin \left(2 \pi f_{v k} t+\varphi_{v k}\right)} \cdot e^{-j 2 \pi v f_{r k-\max } \sin \left(2 \pi f_{r k} t+\varphi_{k}\right)} \cdot e^{j 2 \pi\left(k_{x} x+k_{y} y\right)} d k_{x} d k_{y} \\
& =\sum_{k_{\bar{N}}}^{N} \int_{-\infty}^{\infty} \int_{-\infty}^{\infty} e^{-j 2 \pi f_{v k-\max } \sin \left(2 \pi f_{v k} t+\varphi_{v k}\right)} \cdot e^{-j 2 \pi A k_{x} \sin \varphi_{k}} \cdot e^{-j 2 \pi A k_{y} \cos \varphi_{k}} \cdot e^{j 2 \pi\left(k_{x} x+k_{y} y\right)} d k_{x} d k_{y} \\
& =\sum_{k=1}^{N} \delta\left(\left|x-f_{r k-\max } \sin \varphi_{k}\right|+\left|y-f_{r k-\max } \cos \varphi_{k}\right|-\left|f_{v k-\max }\right|\right)
\end{aligned}
$$

where $k_{x}=v \cos \left(2 \pi f_{r k} t\right), k_{y}=v \sin \left(2 \pi f_{r k} t\right), f_{r k-\max }=4 \pi f_{r k} f_{c} R_{k} / c$, and $f_{v k-\max }=4 \pi f_{v k} f_{c} R_{v k} / c$ are the maximum values of the rotating Doppler and the vibrating Doppler of scattering center $k$, 
respectively. It is obvious that the inverse Radon transform result of the rotating scattering center with vibrating interference is defocused into a circular region. The geometric center of the circular region corresponds to the rotating parameters, and the radius of the circular region is in accord with the maximum value of the vibrating Doppler. In practice, however, the vibration amplitude is not always constant, and the defocusing area is not always circular either.

According to Eq. (9) and the result in Fig. 3b, the inverse Radon transform result still has certain value even though it is severely defocused, and the separation of each scattering center just effectively uses the defocusing inverse Radon transform result. Specifically, the parameter estimation mainly includes six steps:

\section{Rotation frequency estimation}

The rotation frequency can be obtained through periodical detection methods such as autocorrelation operation, spectrum analysis, and cepstrum analysis, and it will not be covered here. This paper chooses the autocorrelation operation in time domain for rotation frequency estimation.

\section{Time-frequency analysis of the echo signal}

Since the performance of the method mainly depends on the time-frequency analysis and the inverse Radon transform, the choice of the time-frequency analysis method is crucial. An excellent time-frequency analysis method should be of high resolutions in time and frequency; besides, cannot have cross terms. This paper chooses the short-time Fourier transform (STFT) in both simulation and experiments (there is a phase error because of the discrepancy in the start time between STFT and the signal and can be compensated during parameter estimation).

\section{Obtain a coarse estimation of the parameters}

Obtain a coarse estimation of the rotation parameters through the defocusing inverse Radon transform result. The coarse estimation can be derived by the positions of the local maximum points or the geometric centers of the defocusing areas. In this paper, the geometric centers of the defocusing areas are used to obtain the coarse estimation. The coarse estimation of Fig. $3 \mathrm{~b}$ is shown in Fig. 4.

4. Picked out the available areas of the time-frequency distribution

Picked out the available areas of the time-frequency distribution that correspond to scattering centers to isolate the time-frequency distributions of each scattering center. The shapes of the available areas are consistent with shapes of the coarse estimation in the previous step, and the Doppler ranges of the available areas depend on the defocusing degrees of the scattering centers. In this step, the available area of each scattering center may overlap with each other, but it will not actually impact the whole method. The available areas of the time-frequency distribution in Fig. $2 \mathrm{~b}$ are shown in Fig. 5. 
Fig. 4 The coarse estimation based on the positions of the geometric centers of the defocusing areas in the inverse Radon transform result in Fig. $3 b$

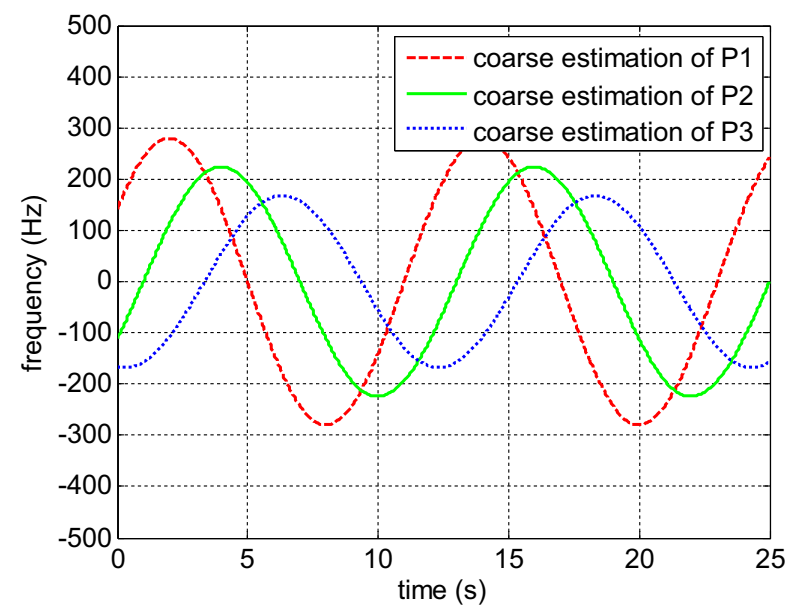

5. Centroid extraction of the time-frequency distributions

Obtain the time-frequency distributions of each scattering center separately and extract their centroid curves. In order to reduce the influence of noise, the following centroid extraction equation is adopted:

$$
\operatorname{centroid}(t)=\frac{\int_{-\infty}^{\infty} f \cdot|T F(t, f)|^{2} d f}{\int_{-\infty}^{\infty}|T F(t, f)|^{2} d f}
$$

The centroid curves of the scattering centers are shown in Fig. 6.

Fig. 5 The available areas of the time-frequency distribution. The time-frequency distribution of each scattering center can be separated by extracting the available areas of the scatter center in Fig. 2b

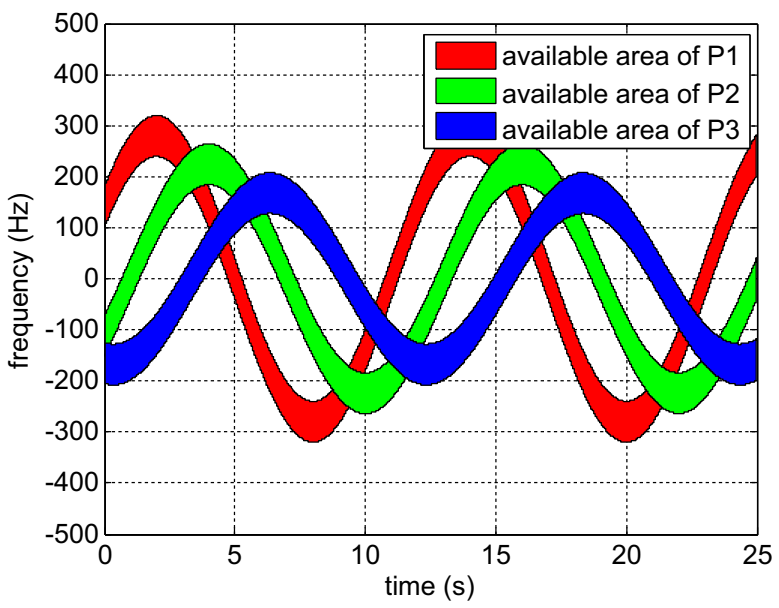


Fig. 6 The centroid curves of the scattering centers

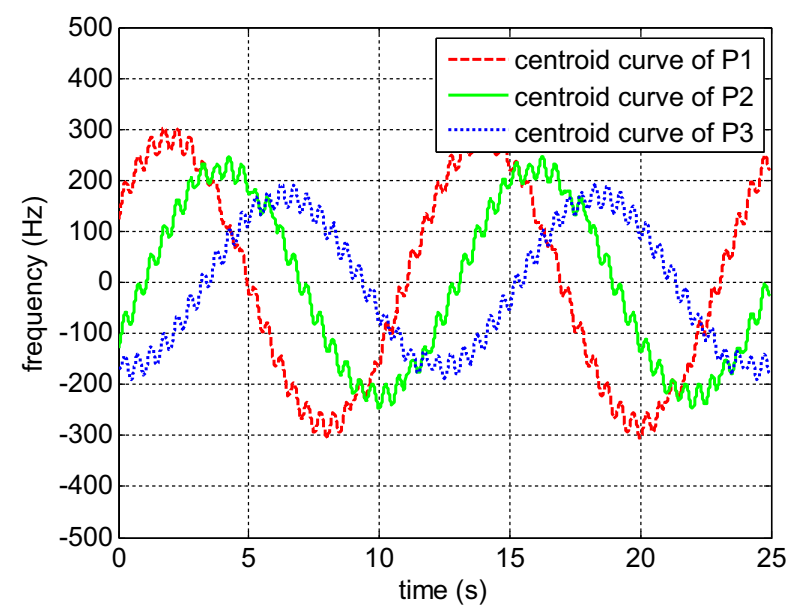

6. Motion separation and parameter estimation

Filter in frequency domain to isolate the rotating Doppler $f_{r d}$ and vibrating interference Doppler $f_{v d}$, as shown in Fig. 7. Estimate the parameters of rotation and vibration, respectively. The estimation results of the simulation scenario well agree with the actual values and demonstrate the correctness and effectiveness of the proposed method.

\subsection{The Image Reconstruction Method}

The inverse Radon transform is a common used image reconstruction method in various areas of science and engineering. It can map sinusoidal curves in the original image to peaks in parameter space according to the image reconstruction theory. Therefore, the inverse Radon transform result in Fig. 3a can also be seen as a reconstructed image while Fig. $3 \mathrm{~b}$ is a defocusing imaging result. In order to obtain high-quality reconstructed images in

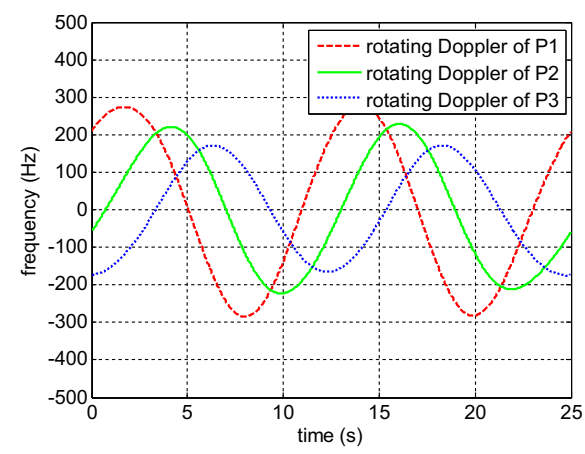

(a) The rotating Doppler

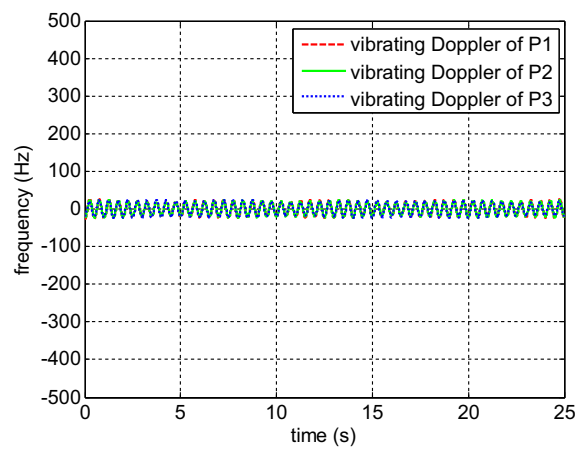

(b) The vibrating Doppler

Fig. 7 The motion separation results based on filtering in frequency domain. a The rotating Doppler. b The vibrating Doppler 
consideration of the vibrating interference, vibration compensation is a necessary processing. The vibration compensation in this paper is mainly based on the time-frequency analysis and its inverse, and image reconstruction is based on the inverse Radon transform. The specific steps are as follows.

1. Obtain the signal of each scattering center

Obtain the signal of each scattering center by the inverse time-frequency analysis of the timefrequency distribution of each scattering center separated in Section 2. Ideally, the signals are as follows.

$$
s_{k}(t)=\sigma_{k}(t) \exp \left(-j \frac{4 \pi f_{c} R_{k}}{c} \sin \left(2 \pi f_{r k} t+\varphi_{k}\right)-\frac{4 \pi f_{c} R_{v k}}{c} \sin \left(2 \pi f_{v k} t+\varphi_{v k}\right)\right), \quad k=1,2 \ldots N
$$

2. Construct the vibrating interference signal

Construct the vibrating interference signal of each scattering center according to the vibrating Doppler $f_{v k}$, as shown below.

$$
s_{v k}(t)=\exp \left(\frac{j}{f_{v k}} \int f_{v k}(t) d t\right), \quad k=1,2 \ldots N
$$

3. Compensate the vibrating interference and reconstruct the rotating signal

Compensate the vibrating interference and reconstruct the rotating signal by conjugate multiplication of the signal of each scattering center in step 1 and the vibrating interference signal of each scattering center in step 2 .

$$
s(t)=\sum_{k=1}^{N} s_{k}(t) \cdot s_{v k}^{*}(t)
$$

4. Time-frequency analysis of the reconstruction signal

Obtain the time-frequency distribution of the reconstruction signal $s(t)$, as shown in Fig. 8. By now, the vibrating interference is already been compensated and the time-frequency curves have reverted to sinusoidal modulation, although it is not perfectly compensated at the junctions of each time-frequency curve.

5. Reconstruct the image of the rotating target

Reconstruct the image of the rotating target through the inverse Radon transform, as shown in Fig. 9. 
Fig. 8 The time-frequency distribution after vibration compensation. It is easy to see that the vibrating interference has already basically been compensated

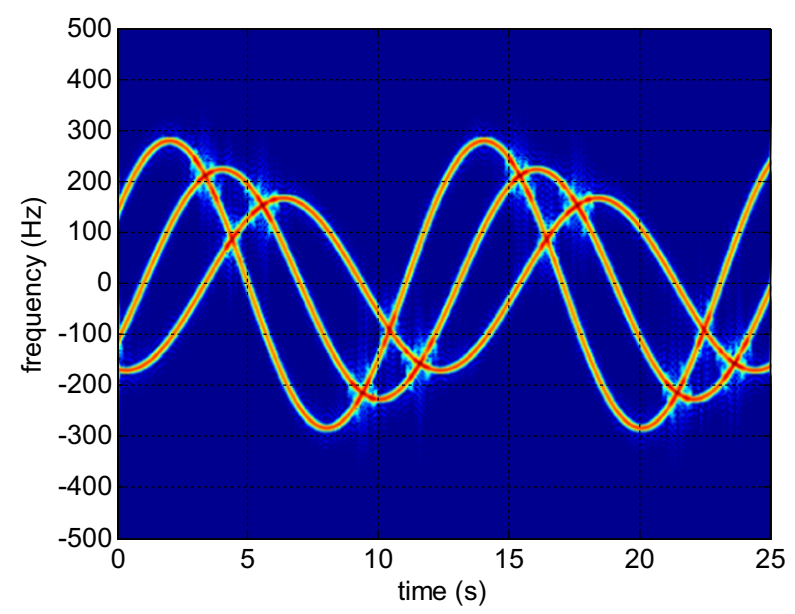

\subsection{The Flow Chart}

To sum up, the flow chart of the whole parameter estimation and image reconstruction processing is shown in Fig. 10.

\section{Experimental Results and Analysis}

\subsection{The Radar System and the Experiments}

A terahertz radar system is established and experiments are carried out to verify the parameter estimation and image reconstruction methods in this paper. The terahertz radar system mainly consists of four modules: the signal source, the RF chains, intermediate frequency (IF) module, and the data collection module. The signal source module consists of a direct digital waveform synthesis (DDWS), a phase-locked loop (PLL), and two coherent local oscillators with a

Fig. 9 The reconstructed image through the inverse Radon transform. Compared with the reconstructed images in Fig. 3b, the focusing degree of the image after vibrating interference compensation is significantly improved, though not as good as Fig. 3a

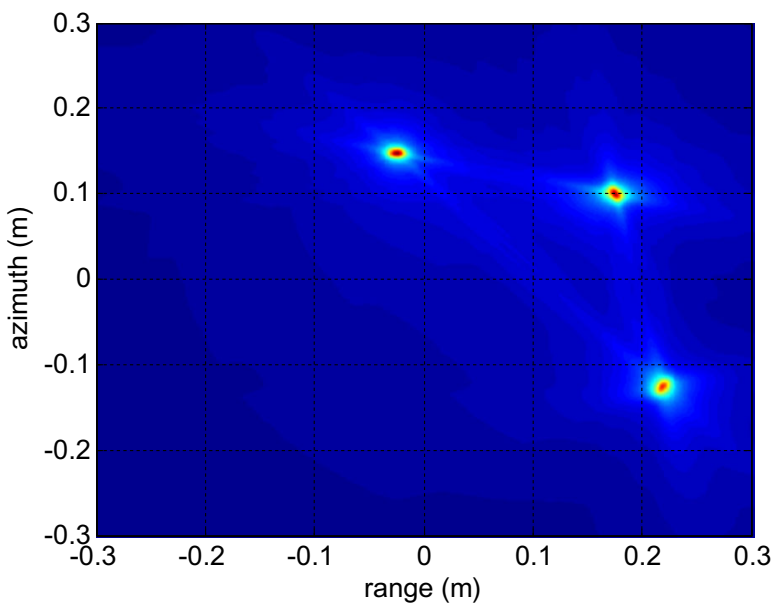




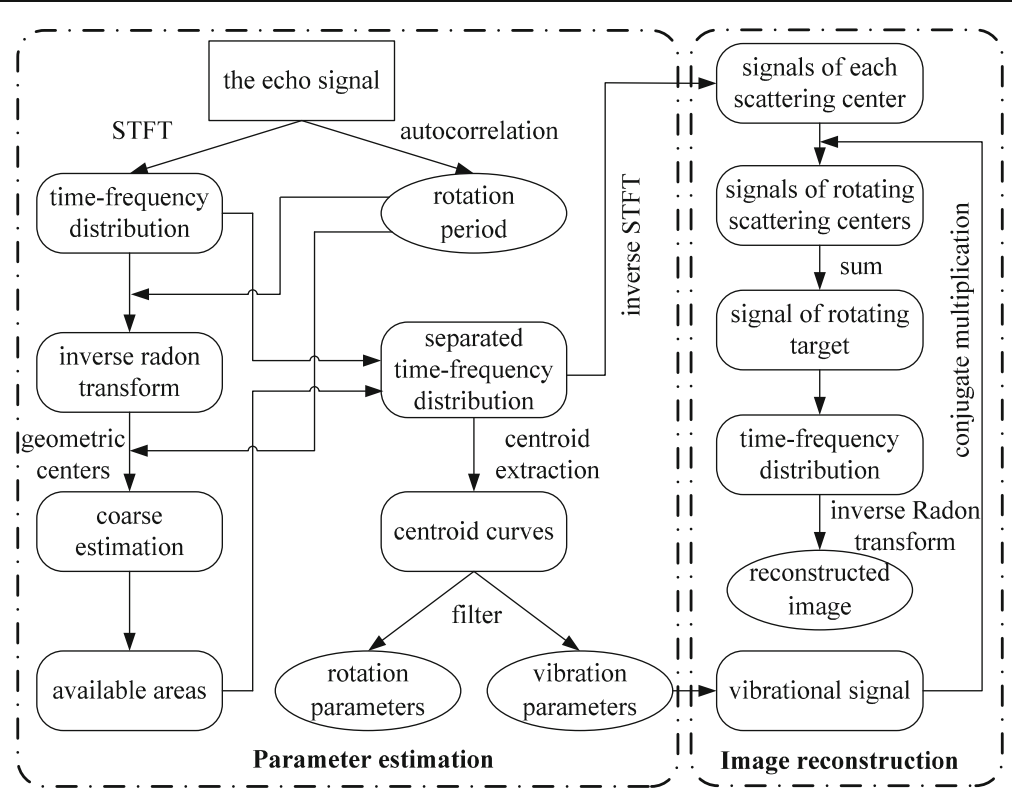

Fig. 10 The flow chart of the processing procedures. It contains all procedures from the echo signal to micromotion parameters and images. The procedures mainly include two parts: parameter estimation and image reconstruction

difference frequency of $60 \mathrm{MHz}$. The PLL can generate signals which have characteristics of broadband, low-spur, and low-phase noise through rapidly changing the frequency-dividing factor [14]. An initial signal range from 2.40 to $3.25 \mathrm{GHz}$ is generated by the DDWS and the PLL and then be modulated onto the coherent local oscillators to output the IF signal varying from 13.40 to $14.2 \mathrm{GHz}$. The mode of the IF signal can be continuous wave $(\mathrm{CW})$, frequencymodulated continuous wave (FMCW), or others. For the sake of micro-motion measurement in this paper, we choose the $\mathrm{CW}$ mode, and the frequency of the IF signal is set to $13.4 \mathrm{GHz}$ because of its relatively high output power.

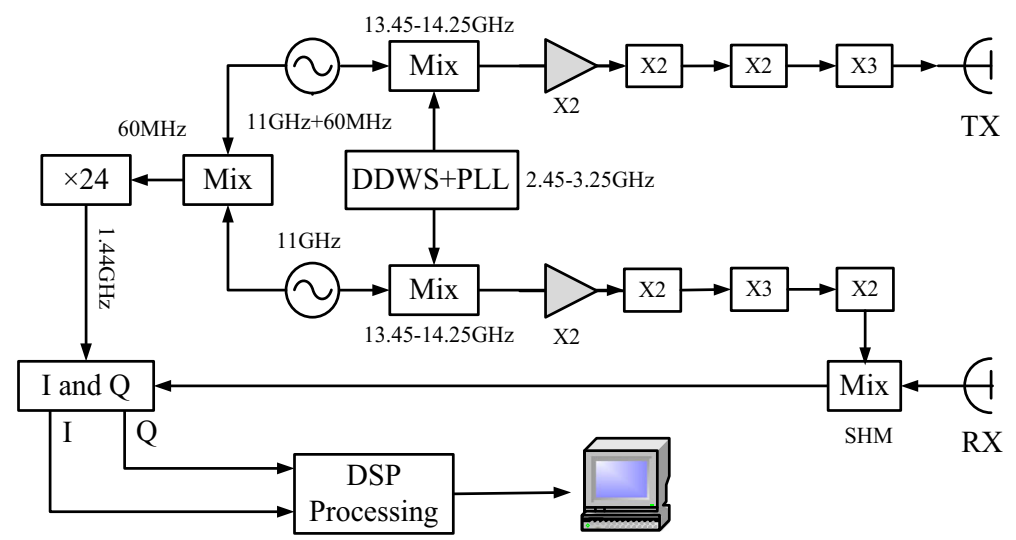

Fig. 11 Schematic diagram of the 322-GHz radar system 
Fig. 12 The transmitting and receiving front-ends of the 322 $\mathrm{GHz}$ radar system

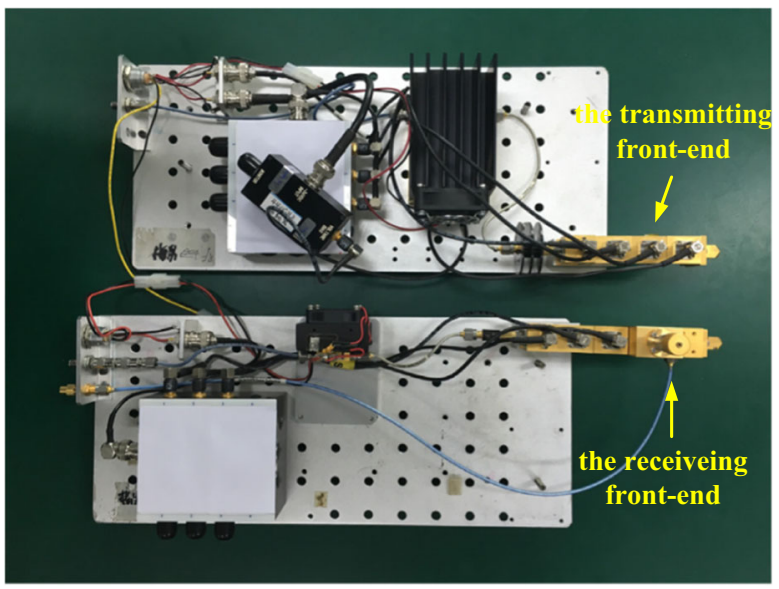

The RF chains are the key design of the terahertz radar system. They consist of the transmitting chain and the receiving chain. In the transmitting chain, the Ku-band IF signal is multiplied into terahertz band after power amplifying and frequency multiplications, and then the terahertz signal is transmitted by a pyramidal horn antenna. Because we set the frequency of the IF signal to $13.4 \mathrm{GHz}$, the carrier frequency of this radar system is about $322 \mathrm{GHz}$. Through harmonic mixing in the receiving chain, the received terahertz signal is down-converted to IF for super heterodyne reception. In order to obtain high sensitivity and large dynamic range of the system, a high-precision controllable digital attenuator with a maximum attenuation of $30 \mathrm{~dB}$ is added to the receiving chain. Through the sub-harmonic mixer (SHM) in the receiving chain, the terahertz signal is down conversed to baseband and demodulated by I/Q demodulator for A/D sampling. The data acquisition and control electronics are based upon a signal processing board with a 14-bit A/D card with a sampling rate of $40 \mathrm{MHz} / \mathrm{s}$. Finally, Ethernet transmits the collected data to the PC for further processing. Both transmitting and receiving signals are controlled by a PC via the PC serial ports and the control software. The schematic diagram of the $322-\mathrm{GHz}$ radar system is shown in Fig. 11, and its transmitting and receiving front ends are shown in Fig. 12.

Fig. 13 The rotating targets. Their essence are two four-sided corner reflectors driving by a motor, and the four-sided corner reflectors can be seen as ideal point targets

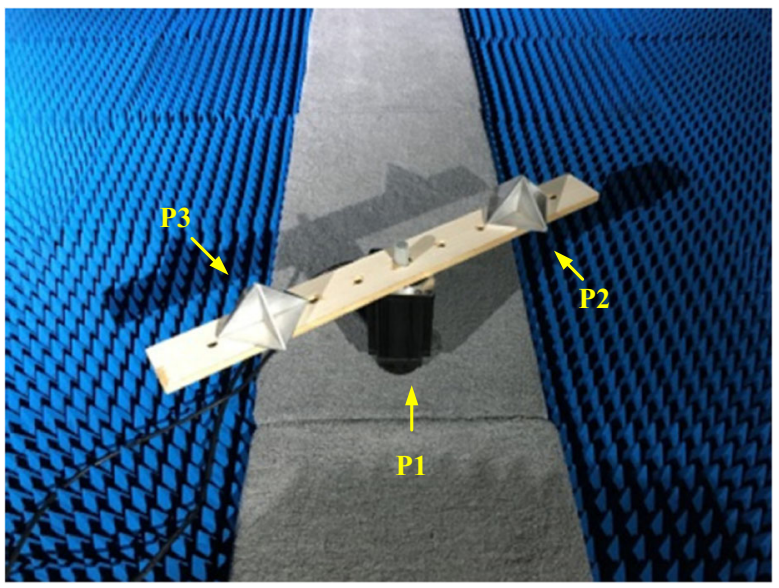


Fig. 14 The time-frequency distribution of the rotating corner reflectors with vibrating interference (322-GHz system)

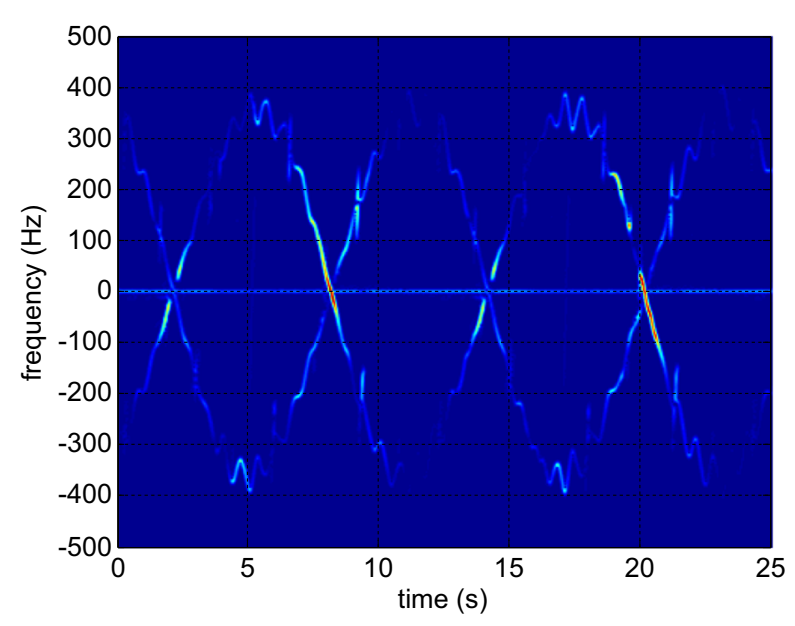

The targets are two rotating corner reflectors driving by a motor (the rotation angular velocities are set to $5 \mathrm{r} / \mathrm{min}$, and the rotation amplitudes are $32 \mathrm{~cm}$ ), and the vibrating interference during the experiments comes from the air movement resulting from an electric fan. All devices are placed in an absorbing chamber to reduce the background noise. The rotating targets are shown in Fig. 13.

\subsection{Parameter Estimation Based on the Experimental Data}

The time-frequency distribution of the echo signal is shown in Fig. 14. The zero-Doppler component comes from the stationary motor, and the other two come from the rotating corner reflectors. It is clear that the micro-Doppler curves of the corner reflectors are distorted because of the vibrating interference.

As it is necessary to know the period of the signal for inverse Radon transform, an autocorrelation operation is made in the first place (Fig. 15). The estimated period is $11.98 \mathrm{~s}$, which is very close to the actual value. The inverse Radon transform result of the

Fig. 15 The period estimation result by autocorrelation

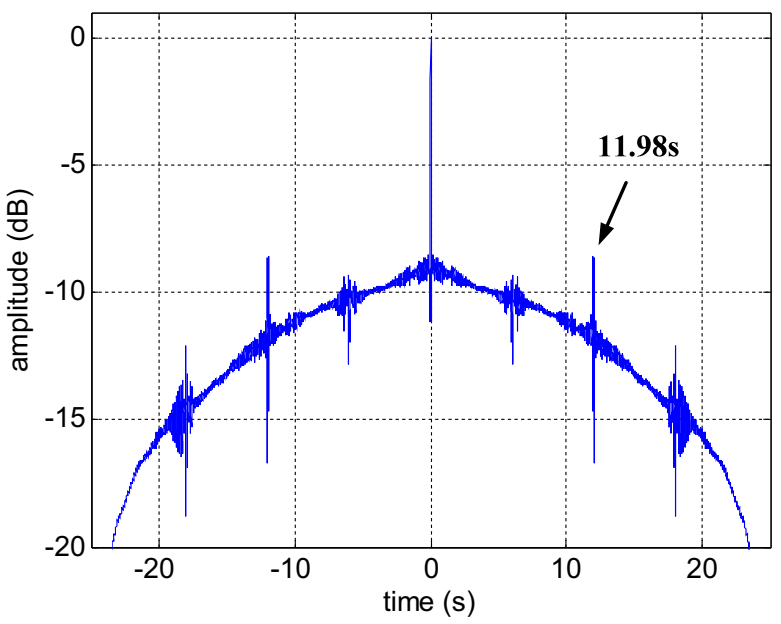


Fig. 16 The inverse Radon transform of the time-frequency distribution in Fig. 14

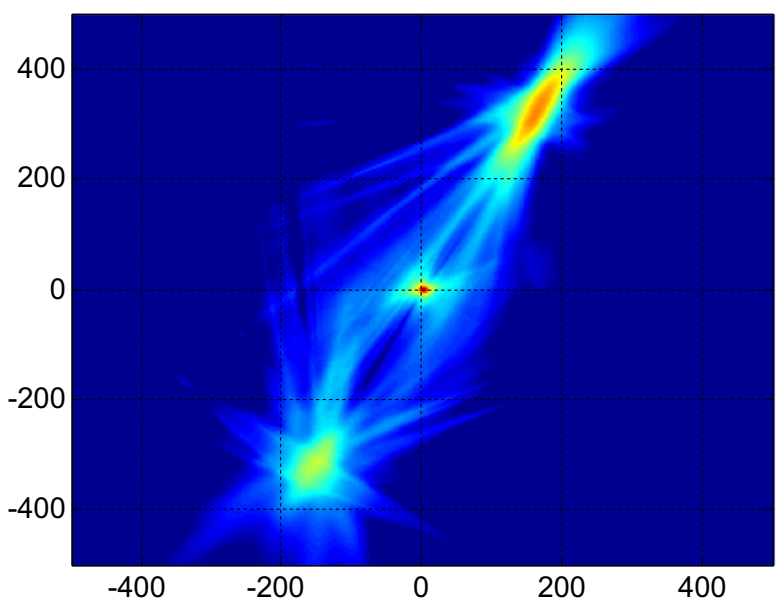

echo signal is shown in Fig. 16. It is pretty obvious that the zero-Doppler component is focused very well after inverse Radon transform, but the rotating components are severely defocused; in addition, the defocusing areas of the corner reflectors are not circular, which indicates that the amplitude of the vibrating interference is not constant. A coarse estimation based on the defocusing inverse Radon transform result is shown in Fig. 17, and the available areas are in Fig. 18. In Fig. 18, the Doppler range of $\mathrm{P} 1$ is set to $10 \mathrm{~Hz}$ due to its good focusing degree; however, the ranges of $\mathrm{P} 2$ and $\mathrm{P} 3$ are set to $150 \mathrm{~Hz}$ in consideration of their defocusing. The separated time-frequency distributions of P1, P2, and P3 are shown in Fig. 19.

After separation of the scattering centers in the time-frequency distribution, centroid curve extraction and motion separation become comparatively easy. The centroid curves through Eq. (10) are shown in Fig. 20, and the rotating Doppler and the vibrating interference Doppler after filtering in frequency domain are shown in Fig. 21.

After obtaining the rotating Doppler and the vibrating interference Doppler, the estimation values of the rotating amplitudes of $\mathrm{P} 1, \mathrm{P} 1$, and $\mathrm{P} 3$ are $0,31.87$, and $31.90 \mathrm{~cm}$. The initial phases are $0^{\circ}, 65^{\circ}$, and $-115^{\circ}$, all of which agree well with the actual values in the experiment.

Fig. 17 The coarse estimation result of the rotating corner reflectors

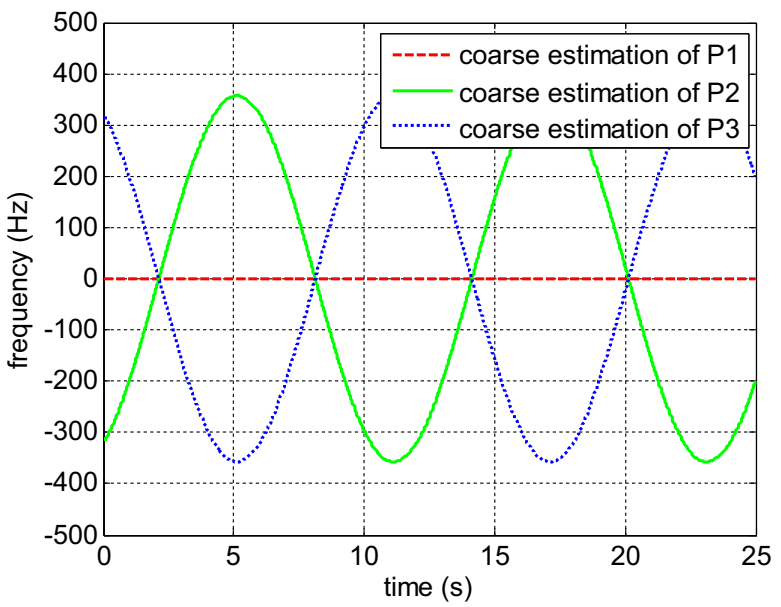


Fig. 18 The available areas of the time-frequency distribution in Fig. 14

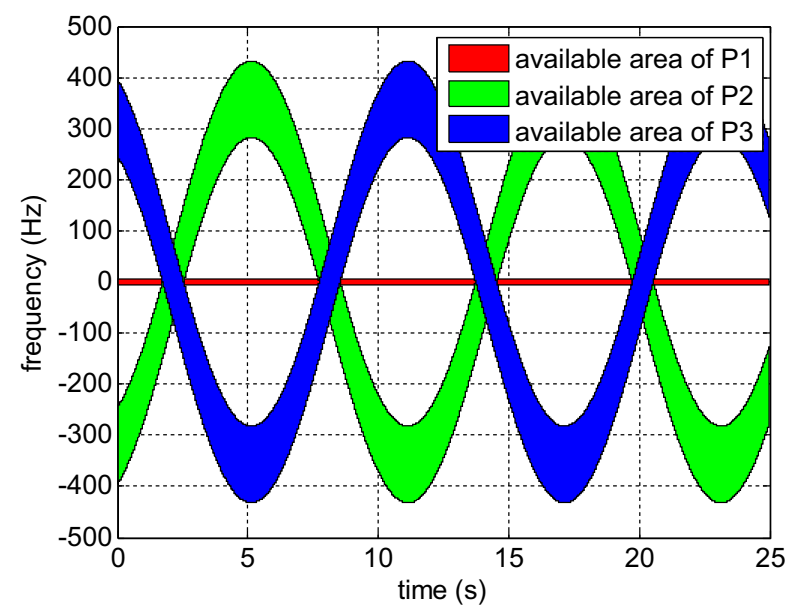

The frequency of the vibrating interference is about $1.5 \mathrm{~Hz}$, and its average amplitude is about $0.87 \mathrm{~mm}$.

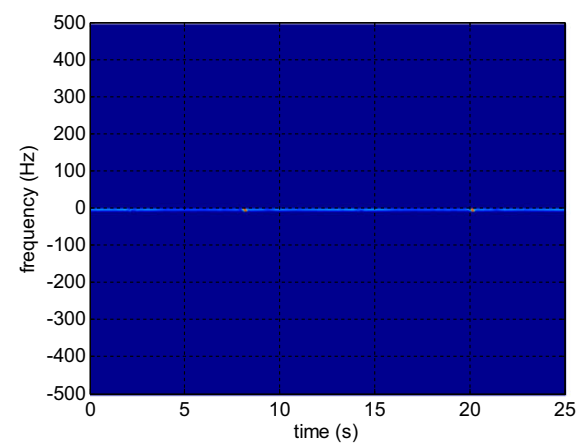

(a) The time-frequency distribution of P1

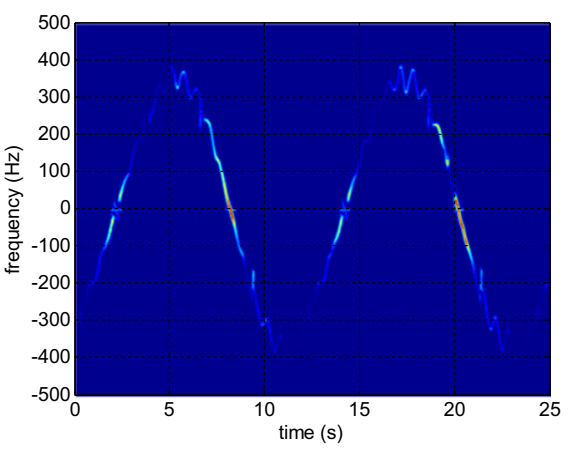

(b) The time-frequency distribution of P2

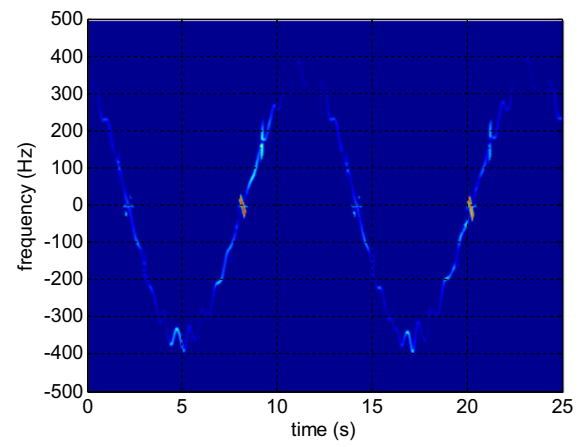

(c) The time-frequency distribution of P3

Fig. 19 The time-frequency distributions of each scatter centers that are separated from Fig. 14. a The timefrequency distribution of P1. b The time-frequency distribution of P2. c The time-frequency distribution of P3 
Fig. 20 The centroid curves of the scattering centers

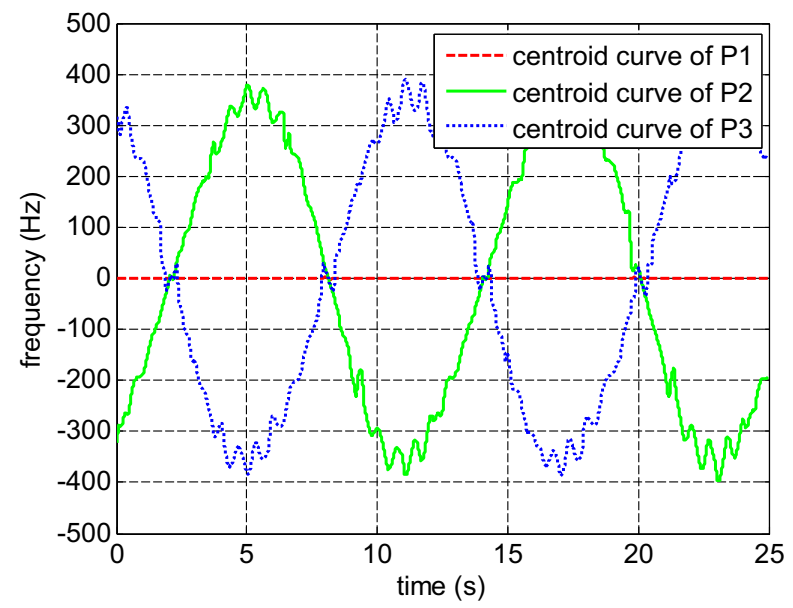

\subsection{Image Reconstruction Based on the Experimental Data}

Since the rotating Doppler and the vibrating Doppler have been separated and estimated, vibration compensation and image reconstruction can be easily conducted by following the steps in Section 3.2. The time-frequency distribution of the reconstruction signal is shown in Fig. 22, and the reconstructed image is shown in Fig. 23. It can be seen from Fig. 22 that the vibrating Doppler has already been compensated basically and the time-frequency curves have come closer to sinusoidal modulation compared with those in Fig. 14. Meanwhile, the reconstructed image in Fig. 23 focuses very well compared with Fig. 16.

\subsection{Experiment Based on a 25-GHz Radar System}

In order to demonstrate the advantages of terahertz radar system in vibrating interference monitoring and micro-motion parameter estimation, a lower frequency commercial radar named SDR Development Kit from ANCORTEK INC is introduced. The kit offers the ability of integrating various software-defined transmitter-receiver systems for detection, tracking,

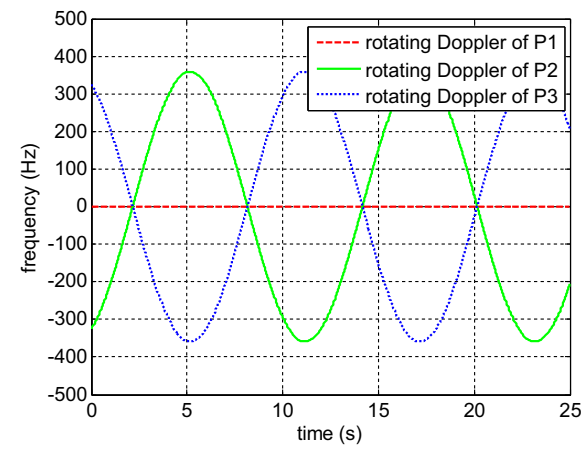

(a) The rotating Doppler

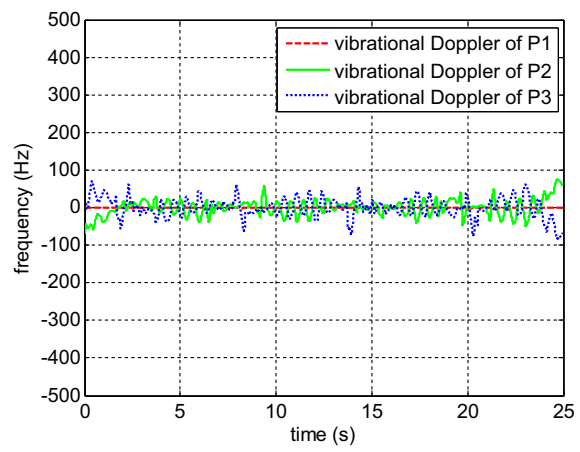

(b) The vibrating Doppler

Fig. 21 The motion separation results based on filtering in frequency domain. a The rotating Doppler. b The vibrating Doppler 
Fig. 22 The time-frequency distribution after vibration compensation

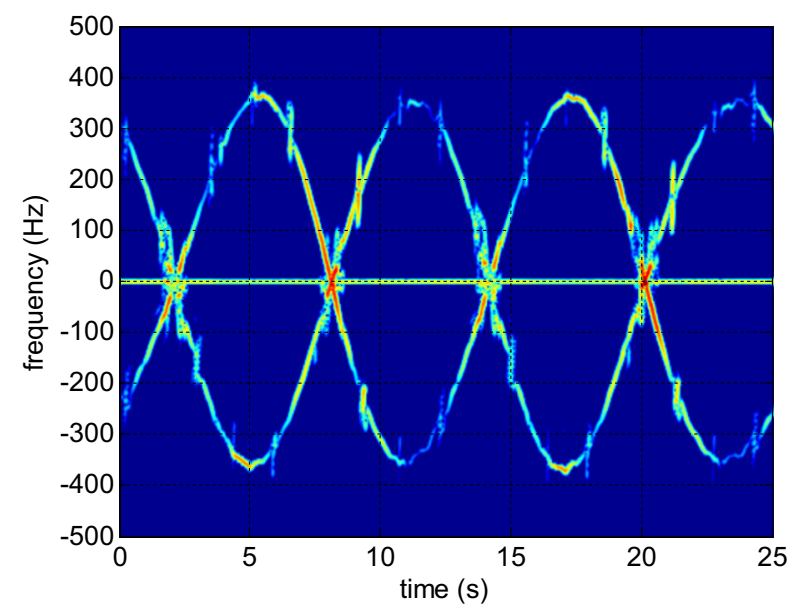

imaging, and measuring of range and Doppler frequency of targets. It has a carrier frequency of $25 \mathrm{GHz}$ and support FMCW, frequency-shift keying (FSK), and CW signal waveforms. The parameter setting and data acquisition are implemented by a PC through the USB 2.0 interface. The $25 \mathrm{GHz}$ Kit and its control and data acquisition PC are shown in Fig. 24.

In order to compare with the terahertz radar system in Section 4.1, we select the CW mode and acquire the data of the rotating corner reflectors with vibrating interference by the kit in the same situation. The time-frequency distribution of the echo signal is shown in Fig. 25. It shows that the Doppler frequency is indeed proportional to the carrier frequency, which can also be inferred from Eq. (4). Therefore, on the one hand, the relatively large Doppler value in the terahertz band provides the possibility for high Doppler resolution and accuracy parameter estimation. For example, there are two scattering centers with a difference Doppler frequency of $1 \mathrm{~Hz}$ at the $25-\mathrm{GHz}$ radar, which is very difficult to be separated from the time-frequency distribution. However, the difference frequency could be magnified to about $13 \mathrm{~Hz}$ to be easily separated at the 322-GHz system. On the other hand, the vibrating interference Doppler which is not prominent at the $25-\mathrm{GHz}$ system is magnified sharply by the same scale, and it will

Fig. 23 The reconstructed image through the inverse Radon transform

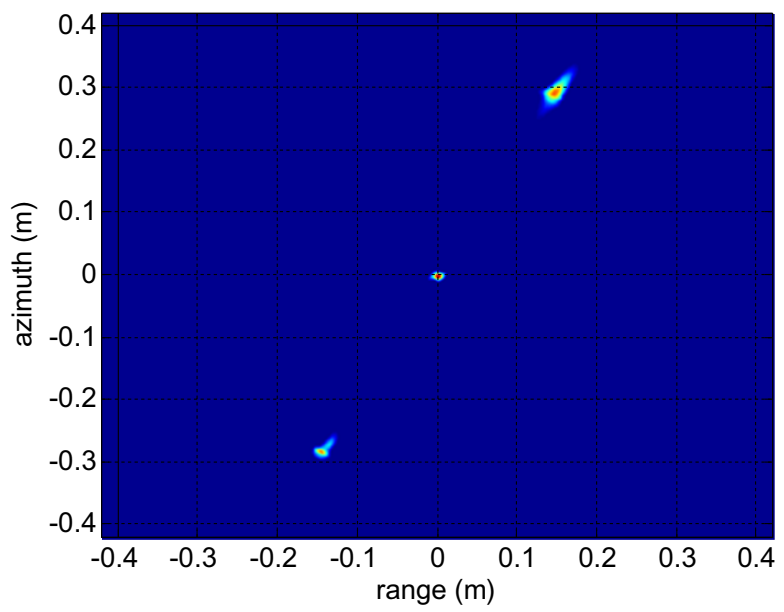


Fig. 24 The $25 \mathrm{GHz}$ Kit and its control and data acquisition PC

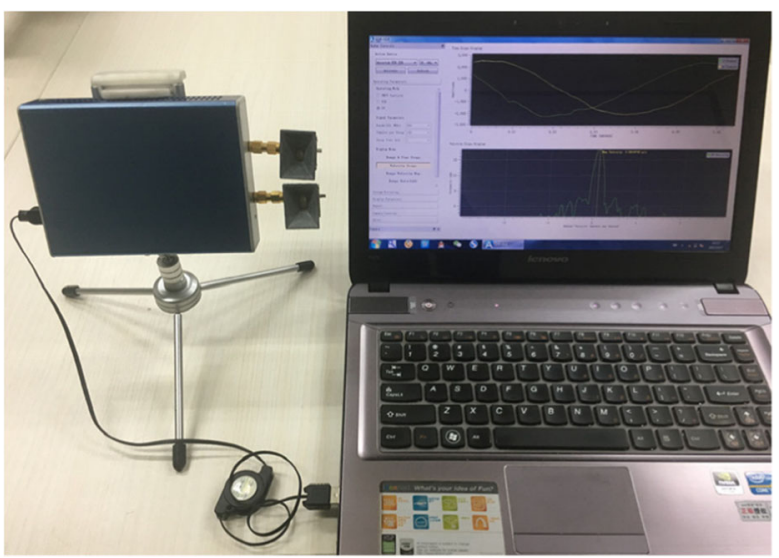

seriously affect the parameter estimation and image reconstruction. But at the same time, this is exactly what we discussed in this paper, and the adverse effect of the vibrating interference Doppler in the terahertz band will be converted to the advantage in vibration monitoring using the method above.

\subsection{Performance Analysis}

The signal-to-noise ratio (SNR) of the echo signal is generally an important factor for parameter estimation and image reconstruction; however, it has a limited impact on the methods proposed in this paper, primarily because of the good noise immunity performance of several operations like the autocorrelation, the centroid extraction and the inverse Radon transform. Based on the Monte Carlo simulation, the relationships between SNR and the parameter estimation errors are qualitatively shown in Fig. 26. The target in simulation is the scattering center P1 in Fig. 1, and the SNR of the echo signal ranges from $-20 \mathrm{~dB}$ to $0 \mathrm{~dB}$. As can be seen from Fig. 26, the SNR of the echo signal seriously affected the accuracy of the

Fig. 25 The time-frequency distribution of the rotating corner reflectors with vibrating interference (25-GHz system)

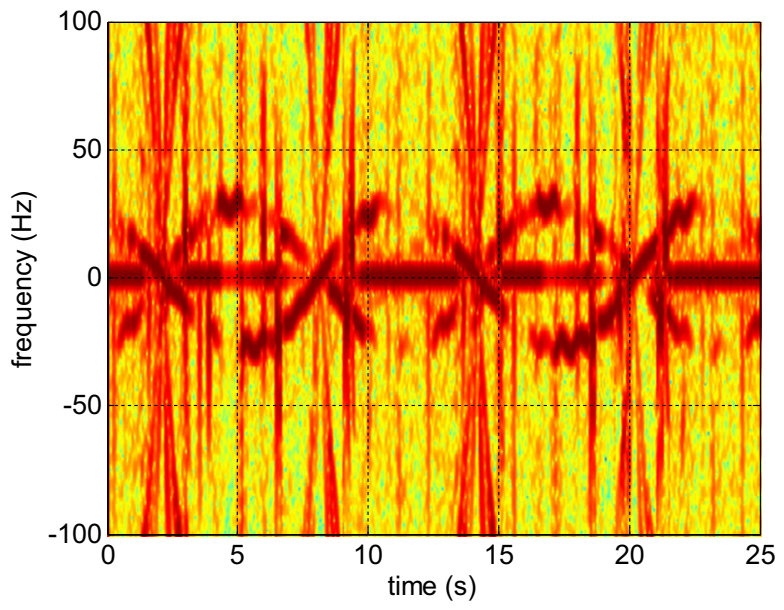


Fig. 26 Relationships between signal-to-noise and the parameter estimation errors

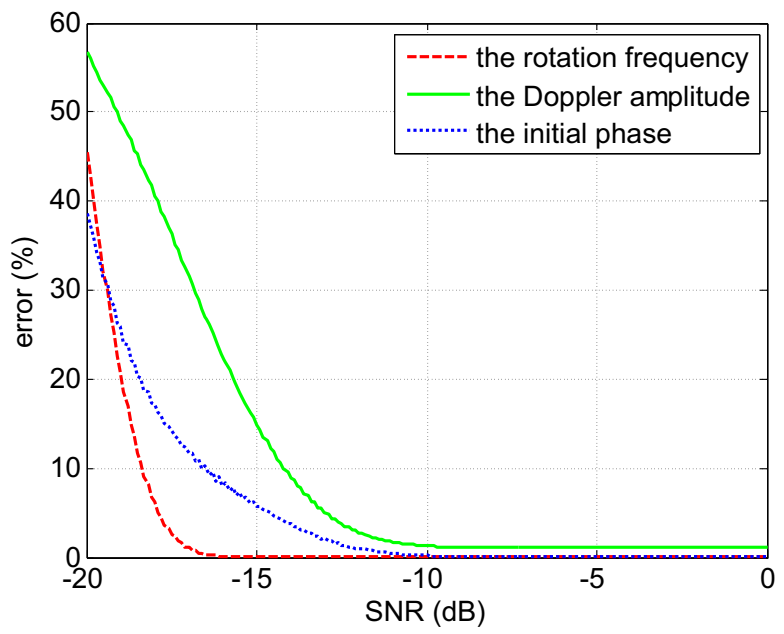

parameter estimation. However, the method proposed in this paper is of good anti-noise ability, especially when the SNR is greater than $-12 \mathrm{~dB}$.

Furthermore, the errors of the parameter estimation will directly affect the vibrating interference compensation and image reconstruction processing, and relationships between errors of the vibrating parameters and the imaging resolution are qualitatively shown in Fig. 27. It appears that the vibrating frequency error plays an important part on the effect of imaging resolution deterioration than the vibrating amplitude error, and the resolution deterioration factor was kept within $3 \mathrm{~dB}$ when the parameter estimation errors were less than $1 \%$, which can be achieved when the SNR is greater than $-12 \mathrm{~dB}$ from Fig. 26. Therefore, the resolution of the reconstructed image is relatively good when the vibrating interference is compensated according to the method proposed in this paper.

Fig. 27 Relationships between errors of the vibrating parameters and the imaging resolution

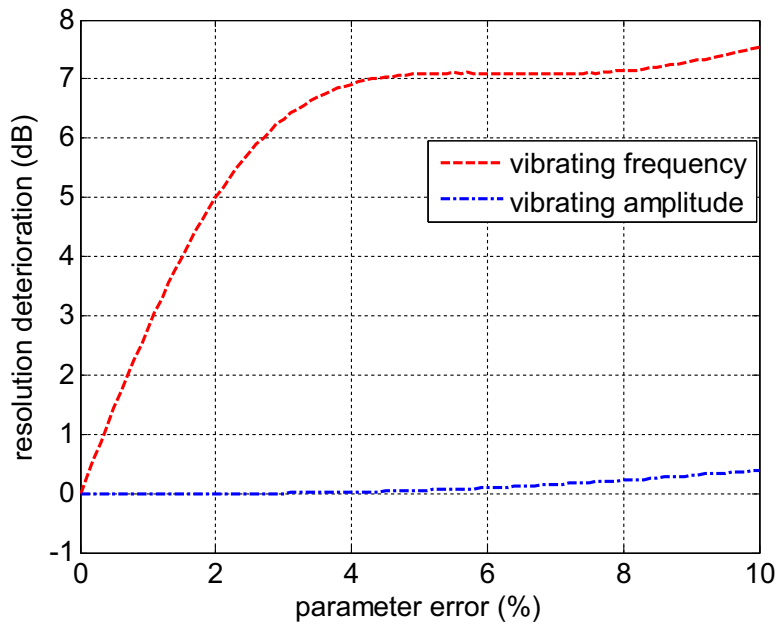




\section{Conclusions}

Parameter estimation and imaging of the rotating targets are of great significance. Meanwhile, the measurement of the vibrating interference while rotating also has an important application value. A parameter estimation method and an image reconstruction method were proposed in this paper, and procedures were given in detail. The parameter estimation method mainly includes steps of time-frequency analysis, inverse Radon transform, the coarse estimation, the centroid extraction, and the filtering in frequency domain, and the rotation and vibrating interference have been separated and estimated accurately in both simulation and experiment. The image reconstruction method consisted of vibrating interference signal reconstruction, vibrating interference compensation, and image reconstruction based on the inverse Radon transform. The high-quality reconstructed images after vibrating interference compensation have verified the validity of the method. The performance of the methods has been proved by the Monte-Carlo simulation results, which fully verify the good performance of the methods in noise immunity. The methods in this paper can also be applied to other micro-motion forms as long as the simple harmonic motion on the radar slight is satisfied.

Open Access This article is distributed under the terms of the Creative Commons Attribution 4.0 International License (http://creativecommons.org/licenses/by/4.0/), which permits unrestricted use, distribution, and reproduction in any medium, provided you give appropriate credit to the original author(s) and the source, provide a link to the Creative Commons license, and indicate if changes were made.

\section{Reference}

1. V. C. Chen, F. Li, SS Ho, et al., Analysis of micro-Doppler signatures, Radar, Sonar, and Navigation, IEEE Proceedings 150 (4), 271-276 (2003).

2. AR Fasih, BD Rigling and RL Moses, Analysis of target rotation and translation in SAR imagery, Proceedings of SPIE - The International Society for Optical Engineering (2009): 7337:73370F-73370F-12.

3. Y. Wang, YC Jiang, Inverse synthetic aperture radar imaging of three-dimensional rotation target based on two-order match Fourier transform, IET Signal Processing 6 (2), 159-169 (2012).

4. F. Santi, M. Bucciarelli and D. Pastine, Target rotation motion estimation from distributed ISAR data, 2012 I.E. Radar Conference (2012), 0659-0664.

5. Y. Zhang, J. Sun, P. Lei, et al., High-frequency vibration compensation of helicopter-borne THz-SAR, IEEE Transactions on Aerospace and Electronic Systems 52 (3), 1460-1466 (2016).

6. Y. Wang, Z. Wang, B. Zhao, et al., Compensation for high-frequency vibration of platform in SAR imaging based on adaptive Chirplet decomposition, IEEE Geoscience and Remote Sensing Letters 13 (6),792-795 (2016).

7. D. Campbell, H. Irwin and J. Rutkoskie, Analysis of vibration effects on mobile missile systems, Guidance, Navigation and Control Conference (1988). doi:10.2514/6.1988-4176.

8. M. Watson, J. Sheldon, S. Amin, et al., A comprehensive high frequency vibration monitoring system for incipient fault detection and isolation of gears, bearings and shafts/couplings in turbine engines and accessories, Power for Land, Sea \& Air 14 (14), 885-894 (2007).

9. J. Li, YM Pi and XB Yang, Micro-Doppler signature feature analysis in terahertz band, Journal of Infrared, Millimeter, and Terahertz Waves 31 (3), 319-328 (2010).

10. ZW Xu, J Tu, J Li, et al., Research on micro-feature extraction algorithm of target based on terahertz radar, EURASIP Journal on Wireless Communications \& Networking 2013 (1), 1-9 (2013).

11. Y. Hua, J. Gao and H. Zhao, The usage of inverse-radon transformation in ISAR imaging, IEEE International Conference on Control Science \& System Engineering (2014).

12. X. Fu, H. Yan, C. Wang, et al., Sequential separation of radar target micro-motions based on IRT, IEEE Transactions on Aerospace \& Electronic System 49 (3), 2073-2083 (2013).

13. X. Bai, F. Zhou, M. Xing, et al., High resolution ISAR imaging of targets with rotating parts, IEEE Transactions on Aerospace and Electronic Systems 47 (4), 2530-2543 (2011).

14. MY Liang, CL Zhang, R Zhao, et al., Experimental $0.22 \mathrm{THz}$ Stepped Frequency Radar System for ISAR Imaging, Journal of Infrared, Millimeter, and Terahertz Waves 35 (9), 780-789 (2014). 